\title{
miR-24-3p Promotes Cell Proliferation, Migration, and Invasion of Human Cervical Cancer Cells through/via Targeting AMOTL2 and Attenuating the YAP/Hippo Pathway
}

\section{Yiyun Huang}

Chongqing Medical University

\section{Lijun Hu}

Chongqing Medical University

Lu Lin

Chongqing Medical University

Yan Liu

Chongqing Medical University

\section{Yan Zhang}

Chongqing Medical University

\section{Lan Zhou}

Chongqing Medical University

He Zha ( $\nabla$ zhahe666@zmu.edu.cn)

The First People's Hospital of Zunyi https://orcid.org/0000-0002-5393-3029

\section{Primary research}

Keywords: AMOTL2, cervical cancer, miR-24-3p, YAP/Hippo signaling pathway

Posted Date: September 9th, 2021

DOI: https://doi.org/10.21203/rs.3.rs-708235/v1

License: (c) (1) This work is licensed under a Creative Commons Attribution 4.0 International License. Read Full License 


\section{Abstract}

\section{Background}

miR-24-3p promotes the development of the majority of malignancies. However, its function in cervical cancer is not clearly elucidated so far.

\section{Methods}

In this study, cell proliferation, migration, and invasion were measured by the CCK8 and transwell assays. Bioinformatic methods were used to predict the target genes of miR-24-3p, verifying by luciferase reporter assay and western blotting. The target genes set was also used for KEGG pathway enrichment analysis.

Results

Then we obsrved higher miR-24-3p level in cervical cancer cells and faster growth of tumor in a xenograft model. The function assays demonstrated that miR-24-3p promoted proliferation, migration, and invasion of cervical cancer cells in vitro. It was confirmed that miR-24-3p directly targeted AMOTL2 and the recovery of AMOTL2 reversed the function of miR-24-3p in cervical cancer cell line CaSki. Besides, miR24-3p suppressed the Hippo signaling pathway in CaSki and SiHa cells.

\section{Conclusions}

In conclusion, our results reminded that miR-24-3p could boost the migration and proliferation of cervical cancer cells via down-regulating AMOTL2 and attenuating YAP/Hippo signaling pathway activity.

\section{Introduction}

Cervical cancer with significant morbidity among female genital malignancies is one of the most common malignancies worldwide ${ }^{1}$. New cases of cervical cancer were approximately 569,847 people and more than half of them died in $2018^{2}$. Although now we have regular screening and prevention strategies, the available treatment schemes still pose a threat to fertility. Recurrent and metastatic cervical cancer is generally incurable and it has a poor the prognosis at the same time. Therefore, it is urgently needed to identify distinctive markers of cervical cancer for ascertaining diagnostic and treatment strategies.

miRNA consisting of roughly 22 nucleotides is a class of endogenous non-coding single-stranded small RNA that can inhibit or activate target genes at the transcriptional or post-transcriptional level ${ }^{3}$, which exerts a siginificant function on proliferation, apoptosis, invasion, and epithelial-mesenchymal transition (EMT) of cancer cells ${ }^{4}$. miR-24-3p as a member of miR-24 family plays an oncogenic role during the development of multiple kinds of tumors ${ }^{5-7}$. For example, miR-24-3p and miR-27a-3p improve proliferation of glioma cells with regulating MXI1 cooperatively ${ }^{8}$. miR-24-3p overexpression promote the migration of MCF7 cells and mediate the tamoxifen resistance in breast cancer ${ }^{9,10}$. No wonder that some miRNAs can exert disputed functions in different cancers, a recent research reveals that miR-24-3p 
decelerates the progression by downregulating LAMB3 in Pancreatic ductal adenocarcinoma (PDAC) ${ }^{11}$. Nonetheless, it remain to figure out the explicit function of miR-24-3p in cervical cancer .

, Angiomotin (AMOT), angiomotin-like 1 (AMOTL1), and angiomotin-like 2 (AMOTL2) make up the motin family, their protein sequences are similar. According to yeast two-hybrid screening, angiomotin was regarded as an angiostatin-binding protein to modulate endothelial cell migration ${ }^{12,13}$. The motin family is also known as AMOTs, functional studies have demonstrated that AMOTs are regulatory factors in the Hippo signaling pathway, they interact with multiple core proteins including Merlin, LATS1/2, MST1/2, and YAP $1{ }^{14}$. Since AMOTL1 and AMOT2 reserve the LPTY motif ${ }^{15}$, they can direct interact with YAP1 through protein-protein mode of action to regulate YAP1 cytoplasm-to-nucleus translocation ${ }^{16}$. YAP1 has been confirmed to promote the proliferative and migrated ability of cervical carcinoma cells ${ }^{17}$. However, few studies have focused on the function of AMOTL2 in human cervical cancer. AMOTL2 has been shown to be a key factor in the normal movement of embryonic cells ${ }^{18}$ and is associated with the migration of endothelial cells ${ }^{19}$. Meanwhile, AMOTL2 is a underlying target of miR-24-3p, as predicated by miRDB, Targetscan, mitarbase, and starbase. Therefore, it is quite interesting to clearly elucidate the relationship among miR-24-3p, AMOTL2, and YAP1 during cervical cancer progression.

In this study, we identified that miR-24-3p is significantly upregulated in $\mathrm{SiHa}$ and CaSki cells and is a critical promoter of cell proliferation, migration, and invasion. Importantly, we demonstrated that miR-24$3 p$ downregulation suppressed proliferation and migration through directly targeting AMOTL2. Moreover, miR-24-3p attenuated the YAP1/Hippo pathway. Overall, our data indicated that miR-24-3p might be a potential target for cervical cancer therapy.

\section{Methods And Materials \\ 2.1 Cell Culture}

Human cervical cancer cell lines CaSki and SiHa were stored in school of laboratory medicine (Chongqing medical university, China), and C33A were purchased from Chinese Academy of Sciences (Shanghai, China). $10 \%$ fetal bovine serum (Biological Industries, Israel) was added to Dulbecco's modified Eagle's medium (DMEM; Hyclone, USA). All cells were cultured in prepared medium and were maintained at $37^{\circ} \mathrm{C}$ in a cell incubator containing $5 \% \mathrm{CO}_{2}$.

\subsection{Cell Transfection}

Small interfering RNA (siRNA) for AMOTL2 were purchased from Biomics (Jiangsu, China). Synthesized NC mimics, NC inhibitors, miR-24-3p mimics, and miR-24-3p inhibitors were purchased from GenePharma (Shanghai, China). Cells were transfected with $100 \mu \mathrm{M}$ mimics or inhibitors for twenty-four hours. Lipofectamine 2000 (Invitrogen; Thermo Fisher Scientifc, USA) was used to enhance the transfection efficiency, then we collected and assayed transfected cells. The siRNA sequences are shown in Table I. 


\subsection{RNA Purification and qRTPCR (quantitative Real-time PCR)}

Total RNA of cells was extracted by TRIzol (Invitrogen; Thermo Fisher Scientifc, USA.) method. Reverse transcription was implemented using PrimeScript ${ }^{\mathrm{TM}}$ RT reagent Kit (TaKaRa, Japan) to obtain cDNA templates, and the subsequent reaction procedures were used: $37^{\circ} \mathrm{C}$ for $15 \mathrm{~min}, 85^{\circ} \mathrm{C}$ for $5 \mathrm{sec}$. Then, qPCR was accomplished with $2 \times$ SYBR Green qPCR Master Mix (Bimake; Shanghai, China). GAPDH was used as internal controls for AMOTL2, ANKRDD1, CYR61, and CTGF, and U6 searved as internal controls for miR-24-3p. The thermocycling conditions of qPCR were utilized: $95^{\circ} \mathrm{C}$ for $1 \mathrm{~min}, 95^{\circ} \mathrm{C}$ for $15 \mathrm{sec}$ maintaining 40 cycles, $55^{\circ} \mathrm{C}$ for $30 \mathrm{sec}$ and $70^{\circ} \mathrm{C}$ for $30 \mathrm{sec}$. We adopted the $2-\Delta \Delta \mathrm{Cq}$ method to calculate the RNA expression level. Primers are shown in Table II.

\subsection{Prediction and Analysis of miRNA Target Genes}

The target genes belonging to miR-24-3p were prognosticated by online prediction software, such as mirtarbase (http://mirtarbase.mbc.nctu.edu.tw/index.php), TargetScan (http://www.targetscan.org/), starBase (http://starbase.sysu.edu.cn/index.php), miRDB (http://www.mirdb.org/), then the intersection of targets were captured with Venn (http://bioinformatics.psb.ujent.be/webtools/Venn/) diagram. In addition, pathogenic genes related to cervical cancer are found in Genecards ( https://www.genecards.org/) and were uploaded to the Metascape (http://metascape.org/) database for KEGG signal pathway analysis. Prediction of LncRNAs regulating miR-24-3p was finished by starBase and DIANA in the Venn diagram.

\subsection{Luciferase Reporter Assays}

To construct luciferase reporter plasmids for AMOTL2, we firstly synthesized the seed region of AMOTL2 containing the wild-type or mutant sequences and cloned them into the pGL6-miR luciferase plasmid (Beyotime; Shanghai, China). Later, we cotransfected miR-24-3p mimics or inhibitors ( $50 \mathrm{nM}$ ) and different luciferase reporter plasmids (400 ng) into HEK293T cells for $48 \mathrm{~h}$ using Lipofectamine 2000, and pRL-SV40 luciferase plasmid selected as Renilla luciferase plasmid was transfected with $10 \mathrm{ng}$ in all group. Renilla and Firefly luciferase activities were detected with Dual Luciferase Reporter Assay System (Promega, USA), which also showed the relative luciferase expression.

\subsection{Cell Proliferation Assay}

Cell Counting Kit-8 (Bimake; Shanghai, China) was utilized to test the cell proliferation on the basis of manufacturer's protocol. 2000 cells were seeded in 96-well plates for $24 \mathrm{~h}$, then the NC inhibitor, NC mimic, miR-24-3p inhibitors or mimics was transfected respectively. 24 and $48 \mathrm{~h}$ later, we added $10 \mu \mathrm{l}$ CCK-8 and cultured the cells for $1 \mathrm{~h}$. Finally, the absorbance was measured at $450 \mathrm{~nm}$. Three independent experiments were conducted.

\subsection{Cell Migration and Invasion Analysis}


We adopted Transwell chambers (Corning, USA) to fulfill the two analyses. In the first place, $600 \mu \mathrm{DMEM}$ was added to the lower chamber and $200 \mu$ serumfree medium with transfected cell were seeded into the upper chamber. After incubation at $37^{\circ} \mathrm{C}$ in a cell incubator containing $5 \% \mathrm{CO}_{2}$, the cells in the upper chambers were removed with a cotton swab meticulously, the membranes were fixed with paraformaldehyde and were cleaned with phosphate buffered saline. Finally, we utilize $0.1 \%$ crystal violet to staine,. For migration assay, the number of transfected cells was $4 \times 10^{4}$, the time for incubation is $24 \mathrm{~h}$, besides, $600 \mu \mathrm{l}$ DMEM with 15\% fetal bovine serum was added to the lower chamber. For invasion assay, the number of transfected cells was $8 \times 10^{4}$, the time for incubation is $48 \mathrm{~h}$, in addition, $600 \mu \mathrm{l}$ DMEM with $20 \%$ fetal bovine serum was added to the lower chamber, and a layer of diluted basement membrane Matrigel (Solarbio; Beijing, China) was precoated on the transwell polycarbonate membrane.

\subsection{Western Blotting}

The proteins were extracted from cervical cancer cells and lysed in RIPA (Radio Immunoprecipitation Assay) buffer. The protein concentration was measured by the BCA Protein Assay Kit. The equal $35 \mu \mathrm{g}$ proteins were separated on $10 \%$ sodium dodecyl sulfate polyacrylamide gels and transferred onto polyvinylidene difluoride (PVDF) membranes. $5 \%$ bovine serum albumin (BSA) dissolved in TBST buffer in order to block the nonspecific binding sites in PVDF membrane. Then, the PVDF membranes were incubated with a primary antibody in Primary Antibody Dilution Buffer overnight at $4^{\circ} \mathrm{C}$, membranes were washed with TBST three times for $30 \mathrm{~min}$. Next secondary antibodies were incubated with the membrane for $1 \mathrm{~h}$ at room temperature, and the membranes were washed three times in the same way.

Electrochemical luminescence signals were detected using the Supersignal West Pico Chemiluminescent substrate kit (Millipore, Billerica, MA, USA). Primary Antibody Dilution Buffer, RIPA buffer, and BCA Protein Assay Kit were purchased from Beyotime Institute of Biotechnology (Shanghai, China).

The fowolling antibidies were used: AMOTL2 (1:500, Santa Cruz Biotechnology, CA, USA), p-YAP1 (1:500; Bioss, Beijing, China), YAP1 (1:1000; Bimake, Shanghai, China), and $\beta$-actin (1:500; Affinity Biosciences, USA). HRP-conjugated anti-rabbit or -mouse secondary antibody (1:5000; Beijing Zhongshan Golden Bridge Biotechnology; Beijing, China).

\subsection{Immunohistochemistry}

For IHC analysis, Paraffin-embedded tumor tissue was deparaffinized and the antigen of tumor tissue was retrieved by citric acid buffer (PH6.0). Primary antibodies against p-YAP1 (Bioss, Beijing, China), pLATS1/2 (Affinity Biosciences, USA), and AMOTL2 (Cusabio, China) were diluted at 1:100 and were incubated with the antigen of tumor tissue overnight at $4^{\circ} \mathrm{C}$, then secondary antibodies were incubated with the sections, horseradish peroxidase (HRP) labeled streptomycin solution played a amplification role, the sections were finally reacted with the chromogenic agents. Immunohistochemistry PV-9000 kit (Beijing Zhongshan Golden Bridge Biotechnology; Beijing, China) was utilized for all IHC analyses.

\subsection{Xenograft Model}


The Institutional Animal Care and Use Committee of Chongqing Medical University granted the implmentation of animal studies. We chose 4- 6 weeks old female athymic BABL/c nude mice. CaSki cells were transfected with NC antagomir $(100 \mu \mathrm{M})$ or miR-24-3p antagomir $(100 \mu \mathrm{M})$, respectively. Then $200 \mu$ l phosphate-buffered saline including $4 \times 10^{6}$ CaSki cells were injected subcutaneously into the back flank of nude mice, ten nude mice were divided into two groups randomly. We measured tumor diameters every 7 days for 35 consecutive, next, we taken the tumors from mice and weighted every tumor. The formula of tumor volume was: length $\times$ width $^{2} \times 1 / 2$. The sections of tumor were stained with hematoxylin and eosin.

\subsection{Statistical Analysis}

GraphPad Prism (Prism 5.0, GraphPad Software, San Diego, CA, USA) was used to analyze experimental data, and the student's $t$-test was used to evaluate the differences between two groups. We repeated all the experiments three times, the $p<0.05$ was considered statistically significant. * $p<0.05, * \star p<0.01$, *** $p<0.001$.

\section{Results}

\subsection{Expression of miR-24-3p in cervical cancer cell lines}

In the tumor-miRNA-pathway (http://bioinfo.life.hust.edu.cn/miR_path/index.html) online websites, miR24-3p was shown to be overexpressed in some cancers (Fig. 1A), such as cervical squamous cell carcinoma, endocervical adenocarcinoma (CESC), esophageal carcinoma (ESCA), skin cutaneous melanoma (SKCM), and bladder urothelial carcinoma (BLCA). Moreover, we observed that the mRNA level of miR-24-3p in cervical cancer cell lines $\mathrm{SiHa}$ and CaSki cells was higher than that in cervical cancer cell line C33A cells(Fig. 1B). According to the differential expression of miR-24-3p, it may participate in the development of cervical cancer.

\section{2 miR-24-3p promoted the proliferation, migration and invasion of cervical cancer cells.}

To investigate the biologic function of miR-24-3p in the cervical cancer, we transfected $\mathrm{SiHa}$ and CaSki cells with the miR-24-3p mimic or inhibitor. CCK8 assays showed that downregulating and upregulating of miR-24-3p significantly suppressed and enhanced the proliferation of two cell lines, respectively (Fig. 2A, B). Then we investigated whether the miR-24-3p exerted an influence on the migration and invasion of these cells and found that the migratory and invasive abilities of the two cell lines were markedly enhanced by the miR-24-3p mimic but suppressed by its inhibitor (Fig. 2C, D, E, F). In conclusion, miR-24-3p notably promoted proliferation, migration, and invasion of cervical cancer cells in vitro.

\subsection{AMOTL2 was the direct downstream target of miR-24- $3 p$}


miRNAs regulate a variety of biological processes and cellular activities via regulating their target genes. To select putative targets of miR-24-3p, we adopted a comprehensive in silico analysis with dissimilar miRNA target prediction tools (targetscan, miRDB, and miRtarbase) and discovered a gene set including 95 candidate targets (Fig. 3A). Furthermore, another set consisting of 4 target genes was analyzed by starbase (Fig. 3B); AMOTL2 was the intersection of the two sets and the sequences of conserved 3' untranslated regions ( $3^{\prime}$ UTRs) of AMOTL2 was complementary to miR-24-3p across human, chimp, and rhesus. For further study, original and mutant 3' UTRs of AMOTL2 were cloned into the downstream of coding region in the pGL6-miR luciferase reporter vector, respectively (Fig. 3C).

To ascertain whether AMOTL2 is direct target of miR-24-3p, miR-24-3p mimic or miR-24-3p inhibitor was cotransfected with pRL-SV40 into HEK293T cells. The relative luciferase activity was reduced by $37 \%$ in mimic group or increased by $34 \%$ in inhibitor group in wild-type AMOTL2 3'UTRs-pGL-6 vectors but not in mutant AMOTL2 3'UTRs-pGL-6 vectors (Fig. 3D). miR-24-3p mimics or inhibitors were transfected into SiHa and CaSki cells to identify whether miR-24-3p could reduce AMOTL2 expression, then we discovered that overexpression and inhibition of miR-24-3p contributed to significant reduction and upregulation of AMOTL2 protein level, respectively (Fig. 3E), but the AMOTL2 mRNA level was not changed (Fig. 3F, G). These results suggested that miR-24-3p directly targeted AMOLT2.

\section{4 miR-24-3p promoted the proliferation and migration by targeting AMOTL2}

We utilized small interfering RNAs (siRNAs) of AMOTL2 by knocking down the expression of AMOTL2 to simulate the effects of miR-24-3p overexpression in cervical cancer cells. qRT-PCR and western blotting confirmed the interference efficiency of AMOTL2 in CaSki. As shown in Figs. 4A and 4B, after being transfected with siAMOTL2s, the siRNA3 significantly suspressed the experssion of AMOTL2 and was used in the subsequent experiments. As expected, blocking AMOTL2 expression significantly increased the proliferative (Fig. 4C), migratory, and invasive ability of CaSki cell (Fig. 4D). Next, for proofing whether the revivement of AMOTL2 could rescue the antineoplastic effects of miR-24-3p inhibition, we used CaSki cells co-transfected with miR-24-3p inhibitor and siAMOTL2. Functional assays demonstrated that the suppression of cell proliferation and migration under the miR-24-3p inhibitior were prominently reversed by AMOTL2 down-regulated expression (Fig. 4E, F). Altogether, these data indicated that miR-24-3p exerted carinogenic effect by suppressing AMOTL2 expression in cervical cancer cells.

\section{5 miR-24-3p attenuated the YAP/Hippo signaling pathway}

We found 5003 cervical cancer pathogenic genes in Genecards, 36 of which were candidate targets for miR-24-3p (Fig. 5A). The KEGG signaling pathway enrichment analysis in Metascape showed that these 36 target genes were mainly involved in microRNAs in cancer, leishmaniasis, the ErbB signaling pathway, apoptosis, the signaling pathway regulating pluripotency of stem cells, the phospholipase $D$ signaling pathway, the Hippo signaling pathway, and endocytosis (Fig. 5B). 
It was substantiated that AMOTL2 interacted with the pivotal effector YAP in the Hippo signaling pathway ${ }^{16,20}$. Therefore, we explored the impact of miR-24-3p on the Hippo signaling pathway. It is showed that the downregulation of miR-24-3p in CaSki cells increased levels of phosphorylated-YAP ( $p$ YAP) by western blotting. Instead, overexpression of miR-24-3p in CaSki cells leaded to decreased levels of p-YAP (Fig. 5C). Similar results were observed in SiHa cells. The main downstream targets of YAP included ankyrin repeat domain containing 1 (ANKRD1), connective tissue growth factor (CTGF) and cysteine-rich angiogenic inducer 61 (CYR61). We found that overexpression of miR-24-3p in CaSki cells increased expression of CTGF and Cyr61 at the gene level (Fig. 5D), and downregulation of miR-24-3p in SiHa cells decreased levels of ANKRD1, CTGF, and CYR61 (Fig. 5E). The bioinformatics signaling pathway enrichment analysis results were partly consistent with the experimental results. To sum up, these data indicated that miR-24-3p played a protumor role in cervical cancer through the YAP/Hippo pathway.

\section{6 miR-24-3p promoted tumor growth in tumor-bearing mice}

In order to investigate the contribution of miR-24-3p to the progression of cervical cancer, we implanted CaSki cells transfected with miR-24-3p antagomir or NC antagomir subcutaneously into nude mice, and the growth of the tumors was monitored every 7 days. After 35 days, the tumor volums in miR-24-3p antagomir group were observably smaller than those in NC antagomir group (Fig. 6A, B). In accordance with the tumor volumes, the tumor weights of the miR-24-3p antagomir group were significantly lower than NC antagomir (Fig. 6C). Meanwhile, increased AMOTL2, p-YAP1, and p-LATS1/2 were also found in the miR-24-3p antagomir tumors by IHC (Fig. 6D), which was consistent with the aforementioned results in cells. Thus, our results indicated that miR-24-3p exerted oncogenic effect on the development of cervical cancer in vivo.

\subsection{Prediction and analysis of LncRNAs regulating miR-24- $3 p$}

We used starBase and DIANA to predict LncRNAs binding to miR-24-3p, and the results were shown in Fig. 7A. There were eight LncRNAs as sponges of miR-24-3p, and the names of these LncRNAs were shown in Fig. 7B; The expression of LINC00662, NEAT1, and ILF3-AS1 were low in cervical cancer $(p<$ 0.05). The survival curve analysis of these three LncRNAs in GEPIA showed that the overall survival time of patients with cervical cancer overexpressing ILF3-AS1 was significantly longer than that of those under-expressing ILF3-AS1(Fig. 7F). It was suggested that ILF3-AS1 may act as the sponge of miR-24-3p to regulate AMOTL2 and then inhibit the progression of cervical cancer, which will be the main direction of our follow-up research.

\section{Discussion}

miRNAs can work in a variety of biological processes and participate in the tumor process including uterine cervix carcinoma progression. It has been reported that miR-24 is involved in keratinocyte 
differentiation and its expression is regulated by HPV(Human papilomavirus) $E 6$ and $E 7^{21}$, suggesting that the miR-24 family may be involved in the cervix carcinoma progression. miR-24-3p which is a member of the miR-24 family could inhibit the malignant progression of lacrimal adenoid cystic carcinoma through the p53/p21 signal pathway, but it promoted the development of varied tumors such as breast, lung, liver, and bladder cancer ${ }^{22-26}$. However, the specific role and molecular mechanisms of miR-24-3p, which is a well-known cancer-associated miRNA, have not been elucidated in cervical cancer. In our studies, miR-24-3p was upregulated in SiHa and CaSki cervical cancer cells. Inhibition of miR-24-3p remarkably inhibits cell proliferation, migration, and invasion, but overexpression of miR-24-3p has the opposite effect. Moreover, animal experiments revealed that miR-24-3p antagomir could slow the growth of subcutaneous tumor in nude mice. The above results confirmed that miR-24-3p plays a promoting role in the cervical cancer progression.

One of the biggest challenges in formulating miRNA treatment methods is determining the best miRNA candidate or miRNA target for each disease. Compared to experimental verification, bioinformatics can provide a large variety of high-speed predictions of target genes and related pathways. Four online starbases were used to seek the optimal target gene. The bioinformatics screening results showed that miR-24-3p targets 95 genes in miRDB, mitaRbase, and Targetscan. Biological processes of target genes are enriched in protein autophosphorylation, positive regulation of autophagy, regulation of growth, negative regulation of cell proliferation, DNA damage checkpoint, etc. (Figure S1). Some of these target genes have been linked to cervical cancer; for example, NET1 can promote the angiogenesis of cervical squamous cell carcinoma ${ }^{27}$; likewise, down-regulating ERBB3 of the miR-24-3p target will significantly reduce the proliferation and invasion of cervical cancer cells ${ }^{28}$. AMOTL2 was ultimately selected for validation because it is the intersection in all prediction databases.

AMOTL2 is a member of the Amot family of angiomotin ${ }^{29}$. It has been proven to be a key factor for the normal movement of embryonic cells and is also involved in the migration and proliferation of endothelial cells ${ }^{18,19}$. AMOTL2 plays a role as a pro-oncogene in breast and colorectal cancer ${ }^{30,31}$, but it participates in tumor growth and invasion by suppressing the activation of the target genes of YAP in gliomas as a tumor suppressor gene ${ }^{32}$. According to the existing researches, the function of the AMOTs in cervical cancer remains indistinct, and the precise roles and mechanisms of AMOTL2 in cervical cancer are also elusive. In this reasearch, we definited that AMOTL2 was a functional target of miR-24-3p in uterine cervix carcinoma. First, miR-24-3p negatively modulated AMOTL2 protein levels in cervical cancer cells and in vivo. Second, the activity of a luciferase reporter embedded the 3区-UTR sequence of AMOTL2 was markedly reduced by ectopic miR-24-3p expression but was enhanced by inhibition of miR-24-3p. Third, the restoration of AMOTL2 reversed the antitumor effects of miR-24-3p inhibitors. These observations suggested that AMOTL2 was a direct functional target of miR-24-3p and served as a tumor suppressor in tumorigenesis and the progression of cervical cancer.

To elucidate possible molecular mechanisms modulated by miR-24-3p in cervical cancer, it was discovered that 36 candidate targets were cervical cancer pathogenic genes. Moreover, the KEGG 
signaling pathway enrichment analysis showed that they are mainly involved in microRNAs in cancer, leishmaniasis, the ErbB signaling pathway, apoptosis, the signaling pathway regulating pluripotency of stem cells, the phospholipase D signaling pathway, the hippo signaling pathway, and endocytosis.

Hippo pathway is cognized as a tumor-suppressor pathway negatively modulating the oncoproteins TAZ and YAP ${ }^{33}$. In addition, the Hippo pathway is a conserved signaling cascade and modulate cell growth, homeostasis, fate decision, and regeneration ${ }^{34,35}$. Moreover, the Hippo pathway has recently been associated with the cervical cancer. Hyperactivation of the YAP1 oncogene actuating the initiation and development of cervical cancer was demonstrated by He et al. ${ }^{36}$. Accumulated evidence has highlighted the crucial regulatory function of AMOT family proteins in the Hippo signaling pathway ${ }^{37-39}$. In our study, we further validated that miR-24-3p inactivated the Hippo pathway. Firstly, overexpression of miR24-3p reduced the p-YAP1 level in western blotting, while downregulation of miR-24-3p exerted the opposite effect. Overexpression of miR-24-3p in CaSki cells augmented the mRNA level of CTGF and CYR61 by qRT-PCR. On the contrary, downregulation of miR-24-3p in SiHa cells depressed the mRNA levels of ANKRD1, CYR61, and CTGF. It indicates that miR-24-3p can inhibit the phosphorylation of YAP and activate transcription of target genes downstream of YAP. However, this requires further investigation of the specific mechanism of AMOTL2 on the YAP/Hippo pathway in cervical cancer.

In order to delve into the mechanism of function of miR-24-3p in cervical cancer, we screened out eight LncRNAs that may regulate miR-24-3p by means of bioinformatics. Among them, LINC00662, NEAT1, and ILF3-AS1 were underexpressed in cervical cancer, and the overall survival time of patients with high expression of ILF3-AS1 was longer than that of patients with low expression of ILF3-AS1, and that reminds us that ILF3-AS1 may be a good prognostic marker for cervical cancer, which will provide favorable laboratorial and theoretical bases to subsequent research.

\section{Conclusions}

In summary, this study indicates that miR-24-3p promotes cell proliferation, migration, and invasion in human cervical cancer cells, and it inactivates the AMOTL2/YAP1/Hippo signaling pathway, and the LncRNA ILF3-AS1 may be the underlying upstream regulator of miR-24-3p. It provides novel insight into the molecular basis and may be used as a promising therapy for cervical cancer.

\section{Abbreviations}

EMT

epithelial-mesenchymal transition

PDAC

Pancreatic ductal adenocarcinoma

AMOT

angiomotin

AMOTL1 
angiomotin-like 1

AMOTL2

angiomotin-like 2

siRNA

Small interfering RNA

qRTPCR

quantitative Real-time PCR

RIPA

Radio Immunoprecipitation Assay

PVDF

polyvinylidene difluoride

BSA

bovine serum albumin

BLCA

bladder urothelial carcinoma

CESC

cervical squamous cell carcinoma, endocervical adenocarcinoma

ESCA

esophageal carcinoma

SKCM

skin cutaneous melanoma

p-YAP

phosphorylated-YAP

ANKRD1

Ankyrin repeat domain containing 1

CYR61

cysteine-rich angiogenic inducer 61

CTGF

connective tissue growth factor

\section{Declarations}

\section{Acknowledgments}

Not applicable.

\section{Funding}

This study was supported by the National Nature Science Foundation of China (81760475).

\section{Contributions}


We appreciate our colleagues for their valuable efforts on this article. Yiyun Huang, He Zha, and Lan Zhou designed the research; Lijun Hu and Lu Lin analyzed the data of the database; Yiyun Huang performed all the experiments; Yan Liu performed the xenograft model; Yiyun Huang, He Zha, and Lan Zhou wrote the manuscript.

\section{Ethics declarations}

Ethics approval and consent to participate

All procedures performed in this studies were approved by the Ethics Committee of the Chongqing Medical University.

\section{Consent for publication}

Not applicable.

\section{Competing of Interest}

The authors declare no conflict of interest.

\section{Availability of data and materials}

The data that support the findings of this study are available from the corresponding author upon reasonable request.

\section{References}

1. Siegel RL, Miller KD, Jemal A. Cancer Statistics, 2017. CA: a cancer journal for clinicians. Jan 2017;67(1):7-30.

2. Bray F, Ferlay J, Soerjomataram I, Siegel RL, Torre LA, Jemal A. Global cancer statistics 2018: GLOBOCAN estimates of incidence and mortality worldwide for 36 cancers in 185 countries. CA: a cancer journal for clinicians. Nov 2018;68(6):394-424.

3. Fkih M'hamed I, Privat M, Trimeche M, Penault-Llorca F, Bignon YJ, Kenani A. miR-10b, miR-26a, miR146a And miR-153 Expression in Triple Negative Vs Non Triple Negative Breast Cancer: Potential Biomarkers. Pathology oncology research : POR. Oct 2017;23(4):815-827.

4. Yang N, Ekanem NR, Sakyi CA, Ray SD. Hepatocellular carcinoma and microRNA: new perspectives on therapeutics and diagnostics. Advanced drug delivery reviews. Jan 2015;81:62-74.

5. Sun X, Xiao D, Xu T, Yuan Y. miRNA-24-3p promotes cell proliferation and regulates chemosensitivity in head and neck squamous cell carcinoma by targeting CHD5. Future oncology. Dec 2016;12(23):2701-2712.

6. Wang J, Yin K, Lv X, et al. MicroRNA-24-3p regulates Hodgkin's lymphoma cell proliferation, migration and invasion by targeting DEDD. Oncology letters. Jan 2019;17(1):365-371. 
7. Lin Y, Cao H, Tian Y, Yang X, Zhou C, Zhang Q. miR-24-3p stimulates migration, invasion and proliferation of prostate cancer cells by targeting suppressor of cytokine signaling 6. International journal of clinical and experimental pathology. 2018;11(3):1803-1810.

8. Xu W, Liu M, Peng X, et al. miR-24-3p and miR-27a-3p promote cell proliferation in glioma cells via cooperative regulation of MXI1. International journal of oncology. Feb 2013;42(2):757-766.

9. Oliveto S, Alfieri R, Miluzio A, et al. A Polysome-Based microRNA Screen Identifies miR-24-3p as a Novel Promigratory miRNA in Mesothelioma. Cancer research. Oct 15 2018;78(20):5741-5753.

10. Han X, Li Q, Liu C, Wang C, Li Y. Overexpression miR-24-3p repressed Bim expression to confer tamoxifen resistance in breast cancer. Journal of cellular biochemistry. Aug 2019;120(8):1296612976.

11. Huang W, Gu J, Tao T, Zhang J, Wang H, Fan Y. MiR-24-3p Inhibits the Progression of Pancreatic Ductal Adenocarcinoma Through LAMB3 Downregulation. Frontiers in oncology. 2019;9:1499.

12. Troyanovsky B, Levchenko T, Mansson G, Matvijenko O, Holmgren L. Angiomotin: an angiostatin binding protein that regulates endothelial cell migration and tube formation. The Journal of cell biology. Mar 19 2001;152(6):1247-1254.

13. Bratt A, Wilson WJ, Troyanovsky B, et al. Angiomotin belongs to a novel protein family with conserved coiled-coil and PDZ binding domains. Gene. Sep 18 2002;298(1):69-77.

14. Huang $T$, Zhou Y, Zhang J, et al. The physiological role of Motin family and its dysregulation in tumorigenesis. Journal of translational medicine. Apr 12 2018;16(1):98.

15. Wang C, An J, Zhang P, et al. The Nedd4-like ubiquitin E3 ligases target angiomotin/p130 to ubiquitin-dependent degradation. The Biochemical journal. Jun 1 2012;444(2):279-289.

16. Wang W, Huang J, Chen J. Angiomotin-like proteins associate with and negatively regulate YAP1. The Journal of biological chemistry. Feb 11 2011;286(6):4364-4370.

17. He C, Mao D, Hua G, et al. The Hippo/YAP pathway interacts with EGFR signaling and HPV oncoproteins to regulate cervical cancer progression. EMBO molecular medicine. Nov 2015;7(11):1426-1449.

18. Huang $\mathrm{H}, \mathrm{Lu} \mathrm{Fl}$, Jia S, et al. Amotl2 is essential for cell movements in zebrafish embryo and regulates c-Src translocation. Development. Mar 2007;134(5):979-988.

19. Wang Y, Li Z, Xu P, et al. Angiomotin-like2 gene (amotl2) is required for migration and proliferation of endothelial cells during angiogenesis. The Journal of biological chemistry. Nov 25 2011;286(47):41095-41104.

20. Han H, Yang B, Wang W. Angiomotin-like 2 interacts with and negatively regulates AKT. Oncogene. Aug 10 2017;36(32):4662-4669.

21. Liu F, Zhang S, Zhao Z, et al. MicroRNA-27b up-regulated by human papillomavirus $16 \mathrm{E} 7$ promotes proliferation and suppresses apoptosis by targeting polo-like kinase 2 in cervical cancer. Oncotarget. Apr 12 2016;7(15):19666-19679. 
22. Zhang MX, Zhang J, Zhang H, Tang H. miR-24-3p Suppresses Malignant Behavior of Lacrimal Adenoid Cystic Carcinoma by Targeting PRKCH to Regulate p53/p21 Pathway. PloS one. 2016;11(6):e0158433.

23. Yan L, Ma J, Zhu Y, et al. miR-24-3p promotes cell migration and proliferation in lung cancer by targeting SOX7. Journal of cellular biochemistry. May 2018;119(5):3989-3998.

24. Dong $X$, Ding W, Ye J, et al. MiR-24-3p enhances cell growth in hepatocellular carcinoma by targeting metallothionein 1M. Cell biochemistry and function. Oct 2016;34(7):491-496.

25. Yu G, Jia Z, Dou Z. miR-24-3p regulates bladder cancer cell proliferation, migration, invasion and autophagy by targeting DEDD. Oncology reports. Feb 2017;37(2):1123-1131.

26. Lu K, Wang J, Song Y, et al. miRNA-24-3p promotes cell proliferation and inhibits apoptosis in human breast cancer by targeting p27Kip1. Oncology reports. Aug 2015;34(2):995-1002.

27. Zhang $Y, X i a P, Z$ hang $W$, et al. Short interfering RNA targeting Net1 reduces the angiogenesis and tumor growth of in vivo cervical squamous cell carcinoma through VEGF down-regulation. Human pathology. Jul 2017;65:113-122.

28. Du J, Zhou S, Wang L, Yu M, Mei L. Downregulation of ERBB3 decreases the proliferation, migration and invasion of cervical cancer cells though the interaction with MTK-1. Oncology letters. Sep 2018;16(3):3453-3458.

29. Lv M, Shen Y, Yang J, et al. Angiomotin Family Members: Oncogenes or Tumor Suppressors? International journal of biological sciences. 2017;13(6):772-781.

30. Jiang WG, Watkins G, Douglas-Jones A, Holmgren L, Mansel RE. Angiomotin and angiomotin like proteins, their expression and correlation with angiogenesis and clinical outcome in human breast cancer. BMC cancer. Jan 23 2006;6:16.

31. Mojallal M, Zheng Y, Hultin S, et al. AmotL2 disrupts apical-basal cell polarity and promotes tumour invasion. Nature communications. Aug 1 2014;5:4557.

32. Artinian N, Cloninger C, Holmes B, Benavides-Serrato A, Bashir T, Gera J. Phosphorylation of the Hippo Pathway Component AMOTL2 by the mTORC2 Kinase Promotes YAP Signaling, Resulting in Enhanced Glioblastoma Growth and Invasiveness. The Journal of biological chemistry. Aug 7 2015;290(32):19387-19401.

33. Johnson R, Halder G. The two faces of Hippo: targeting the Hippo pathway for regenerative medicine and cancer treatment. Nature reviews. Drug discovery. Jan 2014;13(1):63-79.

34. Zheng Y, Pan D. The Hippo Signaling Pathway in Development and Disease. Developmental cell. Aug 5 2019;50(3):264-282.

35. Johansen AKZ, Molkentin JD. Hippo signaling does it again: arbitrating cardiac fibroblast identity and activation. Genes \& development. Nov 1 2019;33(21-22):1457-1459.

36. He C, Lv X, Huang C, et al. A Human Papillomavirus-Independent Cervical Cancer Animal Model Reveals Unconventional Mechanisms of Cervical Carcinogenesis. Cell reports. Mar 5 2019;26(10):2636-2650 e2635. 
37. Zhao B, Li L, Lu Q, et al. Angiomotin is a novel Hippo pathway component that inhibits YAP oncoprotein. Genes \& development. Jan 1 2011;25(1):51-63.

38. Kim M, Kim M, Park SJ, Lee C, Lim DS. Role of Angiomotin-like 2 mono-ubiquitination on YAP inhibition. EMBO reports. Jan 2016;17(1):64-78.

39. Paramasivam M, Sarkeshik A, Yates JR, 3rd, Fernandes MJ, McCollum D. Angiomotin family proteins are novel activators of the LATS2 kinase tumor suppressor. Molecular biology of the cell. Oct 2011;22(19):3725-3733.

\section{Tables}

\section{Table I inhibitor/mimic and siRNA sequences}

\begin{tabular}{|ll|}
\hline $\begin{array}{l}\text { inhibitor/mimic and } \\
\text { siRNA }\end{array}$ & Sequences $\left(5^{\prime}\right.$ - $^{\prime}$ ) \\
\hline miR-24-3p inhibitor & CUGUUCCUGCUGAACUGAGCCA \\
\hline NC inhibitor & CAGUACUUUUGUGUAGUACAA \\
\hline miR-24-3p mimic & $\begin{array}{l}\text { Forward:UGGCUCAGUUCAGCAGGAACAG Reverse: } \\
\text { GUUCCUGCUGAACUGAGCCAUU }\end{array}$ \\
\hline NC mimic & $\begin{array}{l}\text { Forward:UUCUCCGAACGUGUCACGUTT Reverse: } \\
\text { ACGUGACACGUUCGGAGATT }\end{array}$ \\
\hline hs-AMOTL2-si-1 & $\begin{array}{l}\text { Forward:GAGAGAUUGGAAUCUGCAAdTdT } \\
\text { Reverse:UUGCAGAUUCCAAUCUCUCdTdT }\end{array}$ \\
\hline hs-AMOTL2-si-2 & $\begin{array}{l}\text { Forward:CAGUACCCUCAUGUUGUACdTdT Reverse: } \\
\text { GUACAACAUGAGGGUACUGdTdT }\end{array}$ \\
\hline hs-AMOTL2-si-3 & $\begin{array}{l}\text { Forward:GUCAGAACAACUGCGAGAGdTdT Reverse: } \\
\text { CUCUCGCAGUUGUUCUGACdTdT }\end{array}$ \\
\hline NC & $\begin{array}{l}\text { Forward:UUCUCCGAACGUGUCACGUdTdT Reverse: } \\
\text { ACGUGACACGUUCGGAGAAdTT }\end{array}$ \\
\hline
\end{tabular}

\section{Table II Primer sequences}




\begin{tabular}{|lll|}
\hline Gene & \multicolumn{2}{l|}{ Primer sequences $\left(5^{\prime}{ }^{\prime}{ }^{\prime}\right)$} \\
miR-24-3p & RT: GTCGTATCCAGTGCAGGGTCCGAGGTATTCGCACTGGATACGACCTGTTC \\
\hline U6 & RT: CGCTTCACGAATTTGCGTGTCAT & \\
\hline miR-24-3p & Forward: GCGTGGCTCAGTTCAGCAG & Reverse: AGTGCAGGGTCCGAGGTATT \\
\hline U6 & Forward: GCTTCGGCAGCACATATACTAAAT & Reverse: CGCTTCACGAATTTGCGTGTCAT \\
\hline ANKRD1 & Forward: CACTTCTAGCCCACCCTGTGA & Reverse: CCACAGGTTCCGTAATGATTT \\
\hline CTGF & Forward: AGGAGTGGGTGTGTGACGA & Reverse: CCAGGCAGTTGGCTCTAATC \\
\hline CYR61 & Forward: AGCCTCGCATCCTATACAACC & Reverse: TTCTTTCACAA GGCGGCACTC \\
\hline AMOTL2 & Forward: TAAAGGTGCTCCATGCCCAG & Reverse: ATCTCTGCTCCCGTGTTTGG \\
\hline GAPDH & Forward: CAGCGACACCCACTCCTC & Reverse: TGAGGTCCACCACCCTGT \\
\hline
\end{tabular}

\section{Figures}

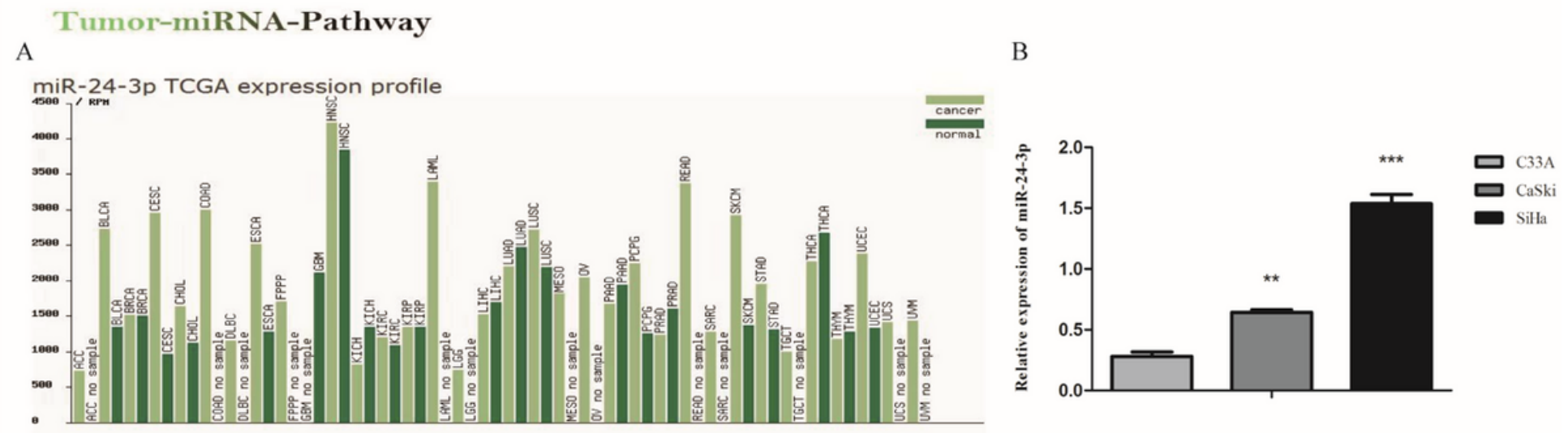

\section{Figure 1}

The expression of miR-24-3p in various cancers and cervical cancer cells. (A) The tumor-miRNA-pathway analysis of miR-24-3p expression in 34 different kinds of cancer. (B) Relative mRNA levels of miR-24-3p in C33A, CaSki, and SiHa cells were measured by qRT-PCR. ${ }^{*} p<0.01,{ }^{* \star *} p<0.001$ vs C33A group. 

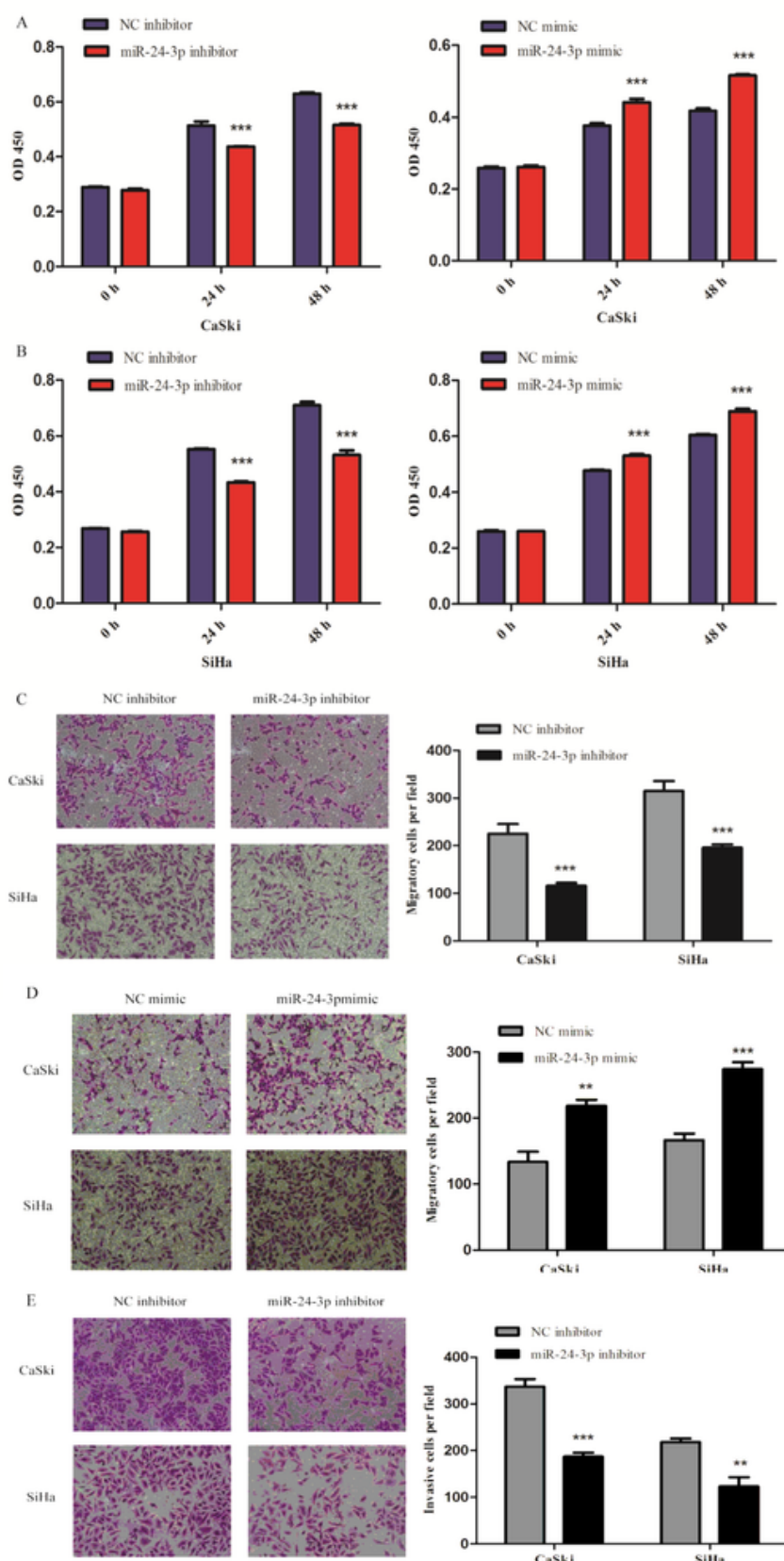

miR-24-3pmimic
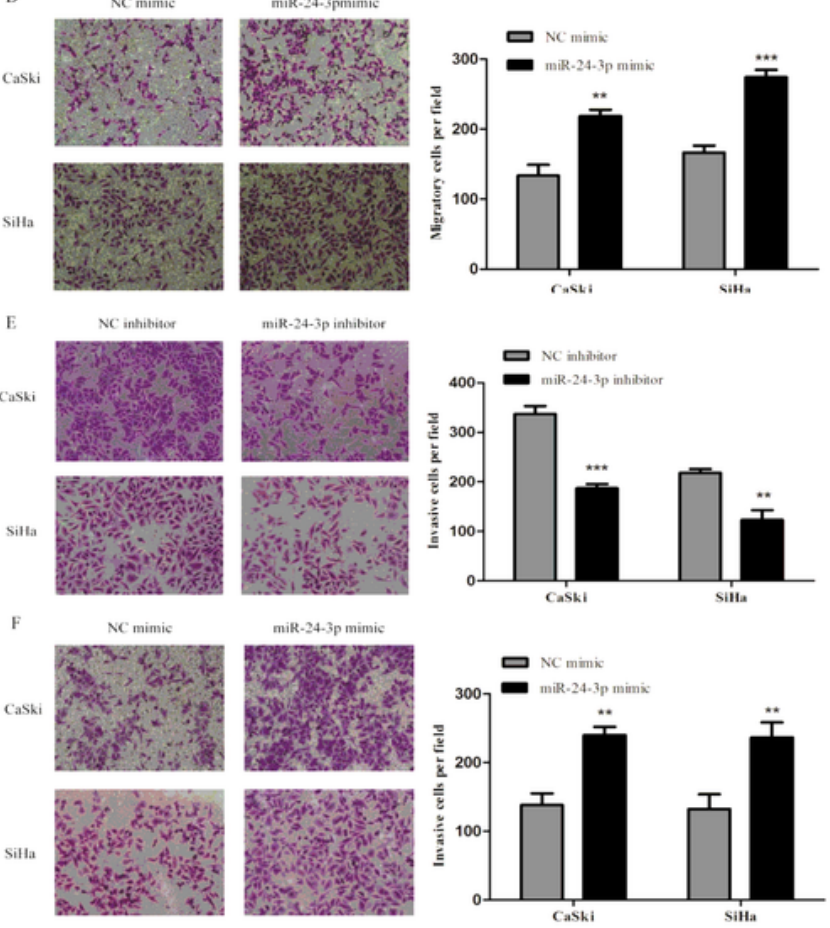

Figure 2

Effect of miR-24-3p on the proliferation, migration, and invasion of CaSki and SiHa cell lines. (A and B) CaSki and SiHa cell lines were transfected with NC inhibitor, miR-24-3p inhibitor, NC mimic, and miR-24-3p mimic, respectively. Cell viability was evaluated using CCK8 assay at the indicated time points after transfection. (C, D, E, and F) Transwell migration and invasion assays were performed after indicated 
transfection in CaSki and SiHa cell lines. The cell numbers of migration and invasion are shown in histograms. ${ }^{\star \star} p<0.01,{ }^{\star \star *} p<0.001$ vs. control groups.

A

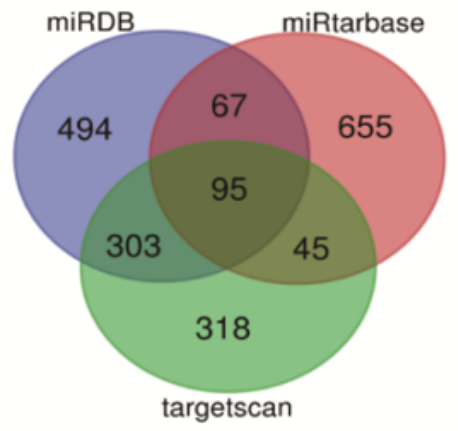

B

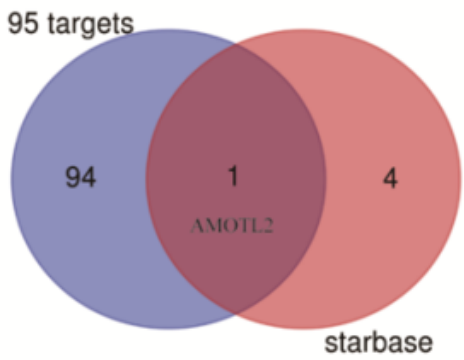

$\mathrm{C}$

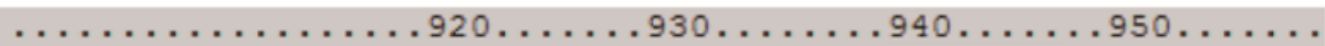
Human --CA---U-C--------CUGUCAGUUUGG-UCAUAAACCCUGAGCCAGAUGAAAUGA-Chimp --CA---U-C-------CUGUCAGUUUGG-UCAUAAACCCUGAGCCAGAUGAAAUGA-Rhesus --CA---U-C--------CUGUCAGUUUGG-UCAUAAACCCUGAGCCAGAUGAAAUGA--

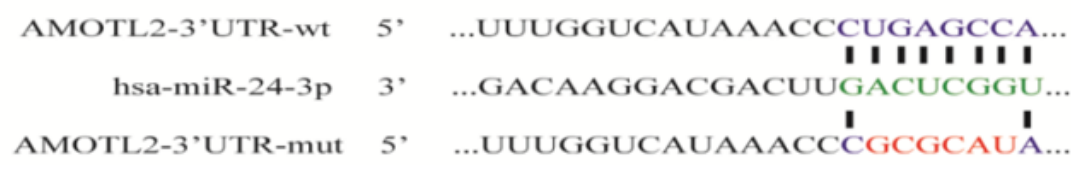

$\mathrm{D}$

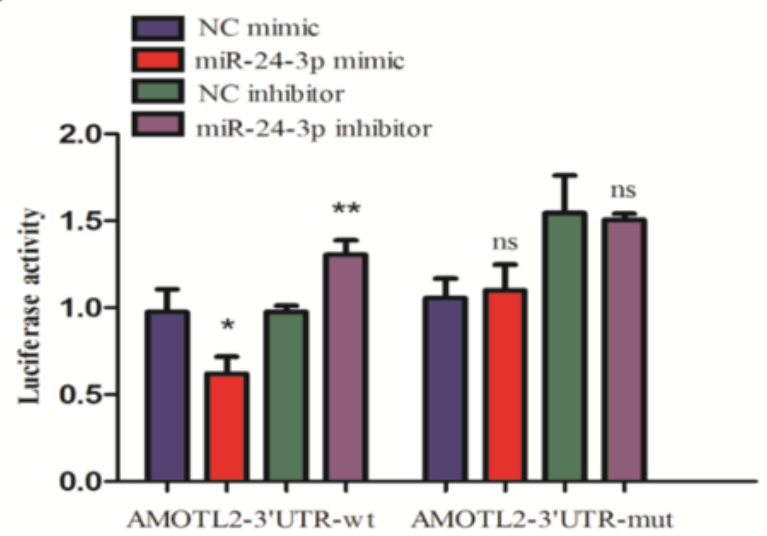

$\mathrm{F}$

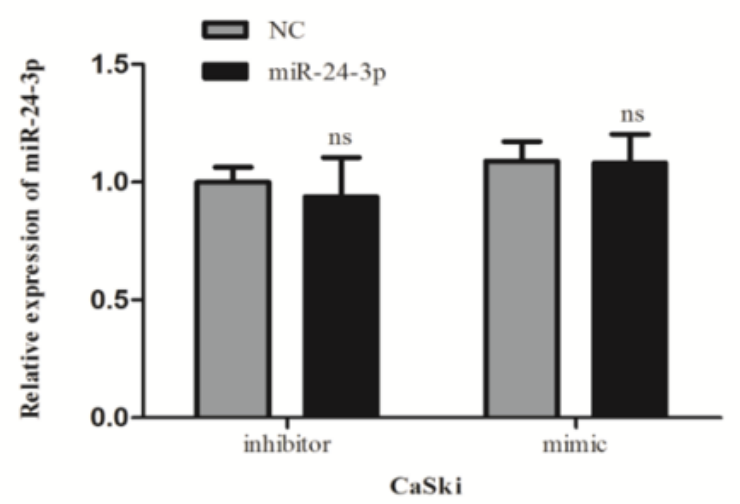

E
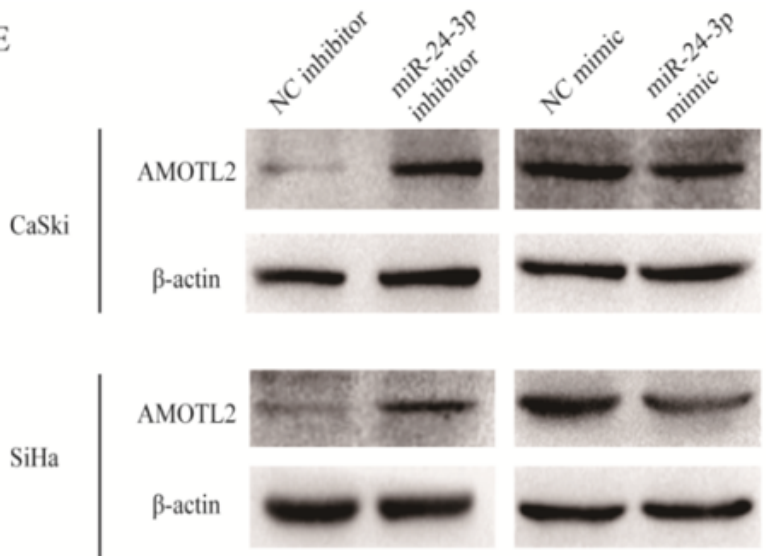

G

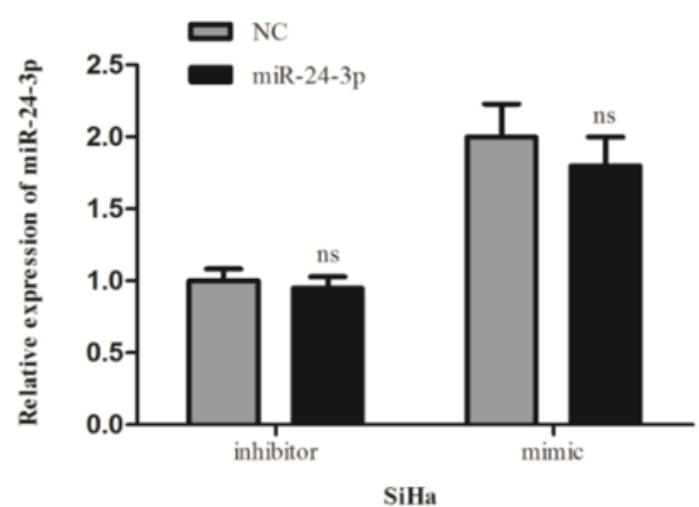

\section{Figure 3}

miR-24-3p directly targeted AMOTL2 3'UTR and suppressed the protein expression of AMOTL2. (A) The Venn diagram indicates the numbers of target genes that overlapped in miRDB, targetscan, and miRtarbase. (B) AMOTL2 was the intersection between aforesaid overlap and predicted targets of 
starbase (programNumヌ7). (C)Upper panel: diagram of miR-24-3p putative seed sequences in the 3' UTR of AMOTL2 mRNA across Human, Chimp and Rhesus. Lower panel: a sketch of construction of wild-type or mutant pGL6-AMOTL2 3' UTR vectors. (D) Dual-luciferase reporter assay was used to analyze the effects of miR-24-3p expression on the activities of wild-type and mutant AMOTL2 3' UTR. (E, F, and G) protein or mRNA levels of AMOTL2 in CaSki and SiHa cells were monitored after miR-24-3p mimic or inhibitor transfection by $q$ RT-PCR and Western blot. ${ }^{*} p<0.05,{ }^{*} p<0.01$ vs. control groups.
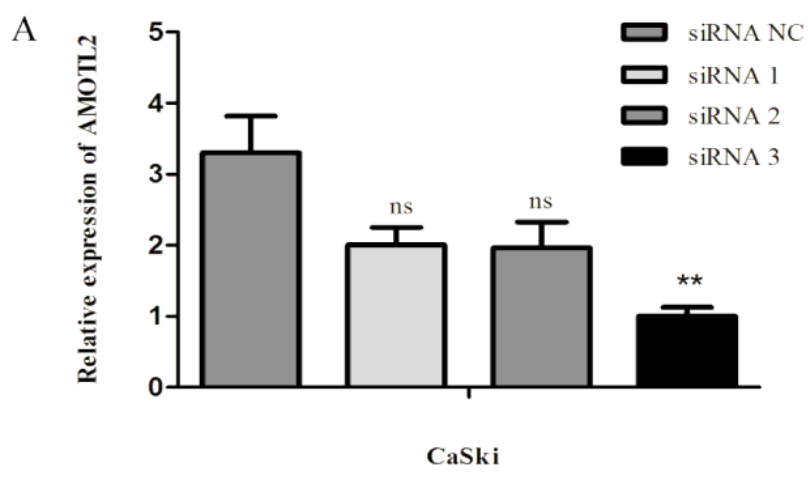

C

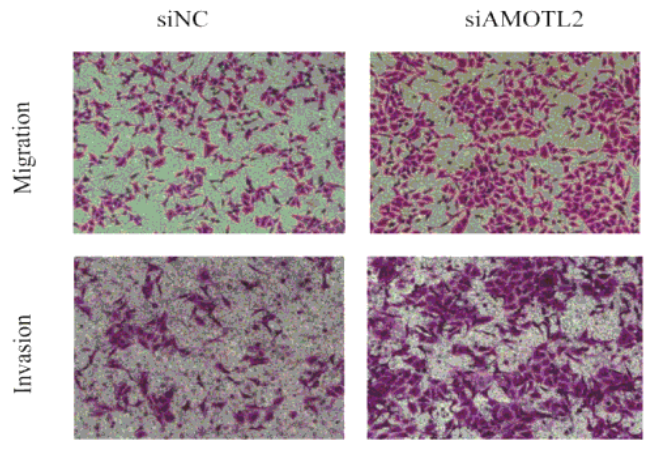

D

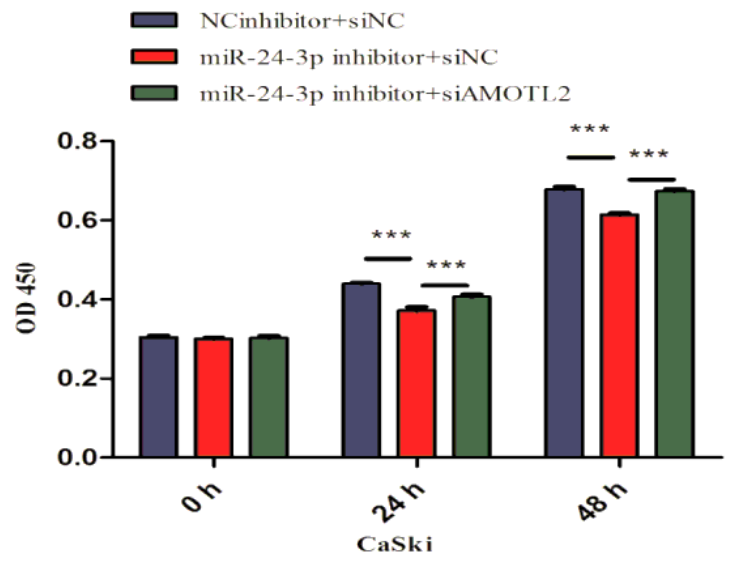

B
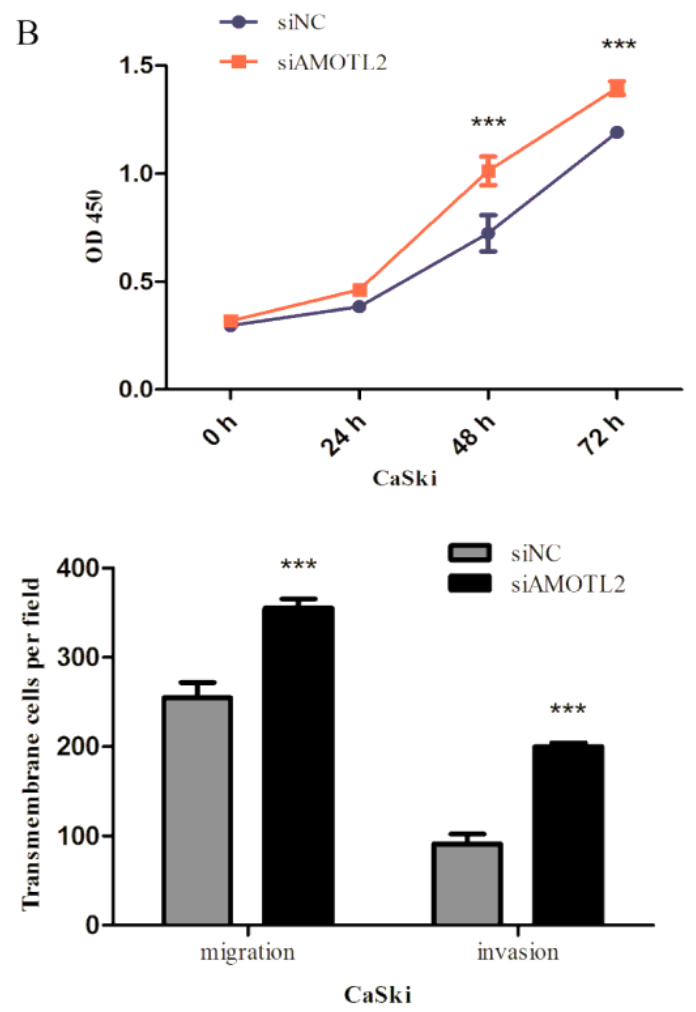

E
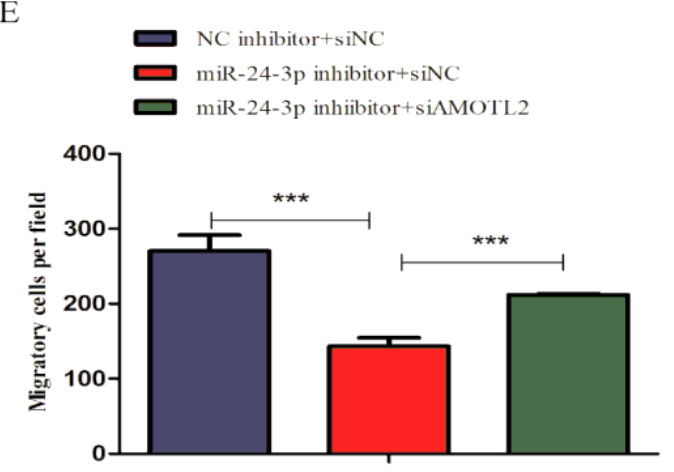

CaSki

miR-24-3p inhibitor NC inhibitor SIAMOTL2 siNC
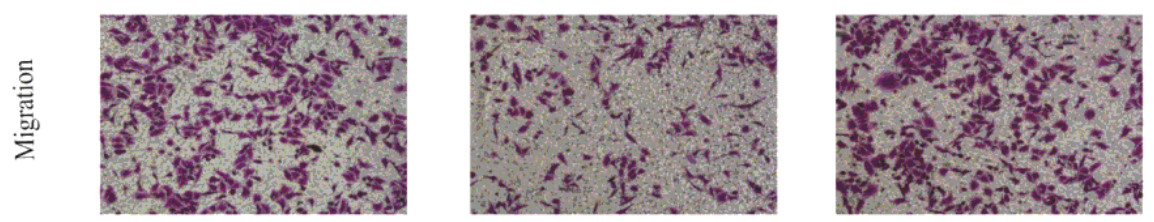

Figure 4 
AMOTL2 knockdown imitated the effects of miR-24-3p overexpression in vitro. (A) The inhibition effect of AMOTL2 siRNAs was verified by qRT-PCR. $(B, C)$ The proliferation of CaSki cells transfected with specific siRNA3 (siAMOTL2) was determined with CCK8 assay (B), then the migration and invasion abilities were assessed using Transwell assays $C)$. (D, E, F) CaSki cells were treated with the indicated reagents and cell viability was analyzed by CCK8 assays (D) and cell migration was determined by Transwell assays (E, F). ${ }^{*} \mathrm{p}<0.01,{ }^{* * *} \mathrm{p}<0.001$ vs. control groups.

A

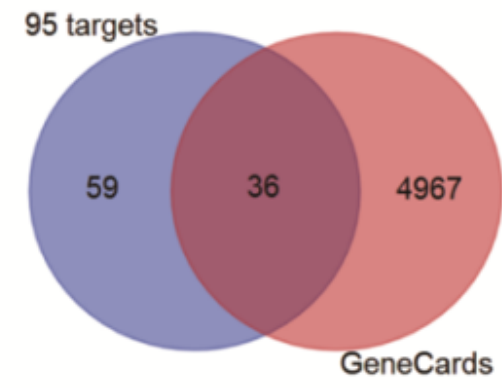

$\mathrm{C}$

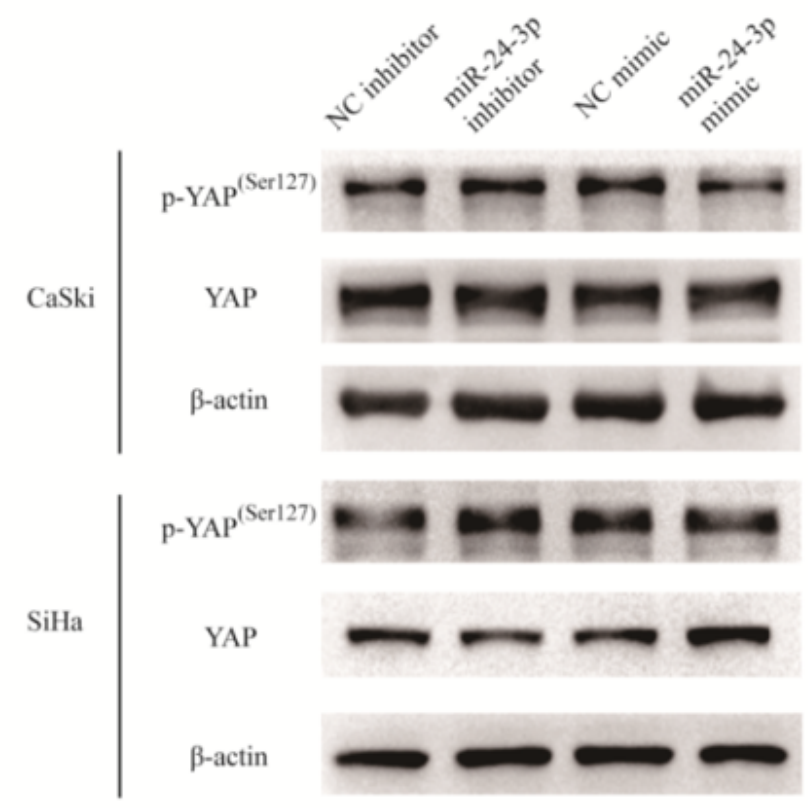

B

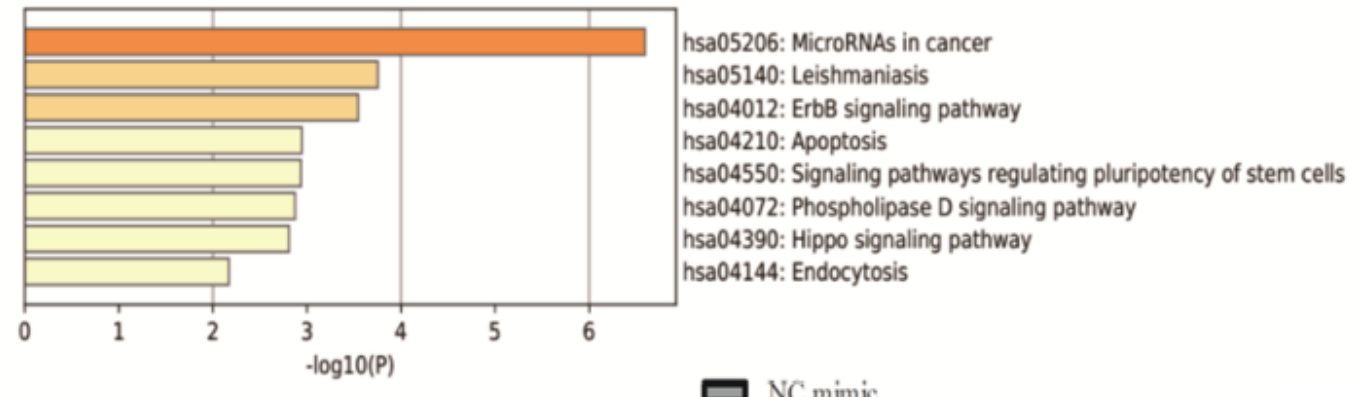

$\mathrm{D}$

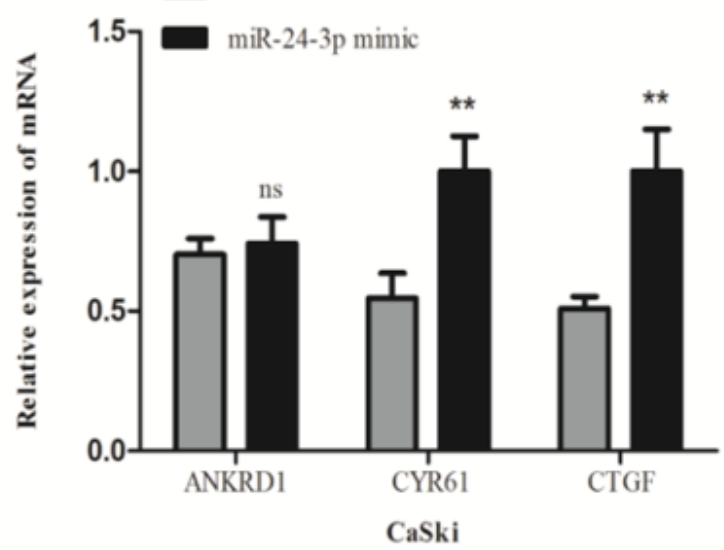

$\mathrm{E}$

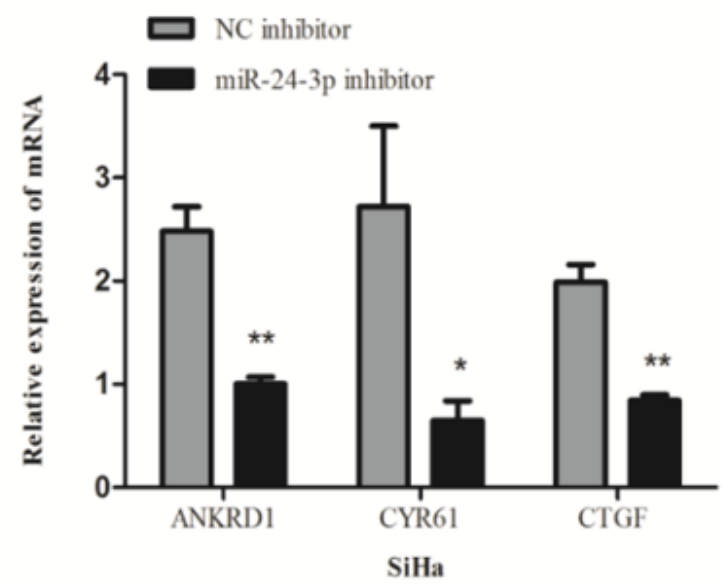

\section{Figure 5}

miR-24-3p inhibited the YAP/Hippo signaling pathway. (A) The Venn diagram indicates the numbers of targets that overlapped in the intersection of 95 target genes and cervical cancer pathogenic genes in Genecards. (B) The KEGG signaling pathway enrichment analysis of 36 target genes in figure A. (C) The CaSki and SiHa cells were transfected with miR-24-3p inhibitor or mimic for $48 \mathrm{~h}$, and then $\mathrm{p}$ - 
YAP(Ser127), YAP and $\beta$-actin levels were determined using Western blot. (D, E) The CaSki were transfected with miR-24-3p mimic (D) and SiHa cells were transfected with miR-24-3p inhibitor (E), relative mRNA levels of YAP target genes, CTGF, CYR61, and ANKRD1 were measured by qRT-PCR. ${ }^{*} p<0.05,{ }^{* *} p<0.01$ vs. control groups.

A

$\mathrm{NC}$ antagomir

miR-24-3p

antagomir
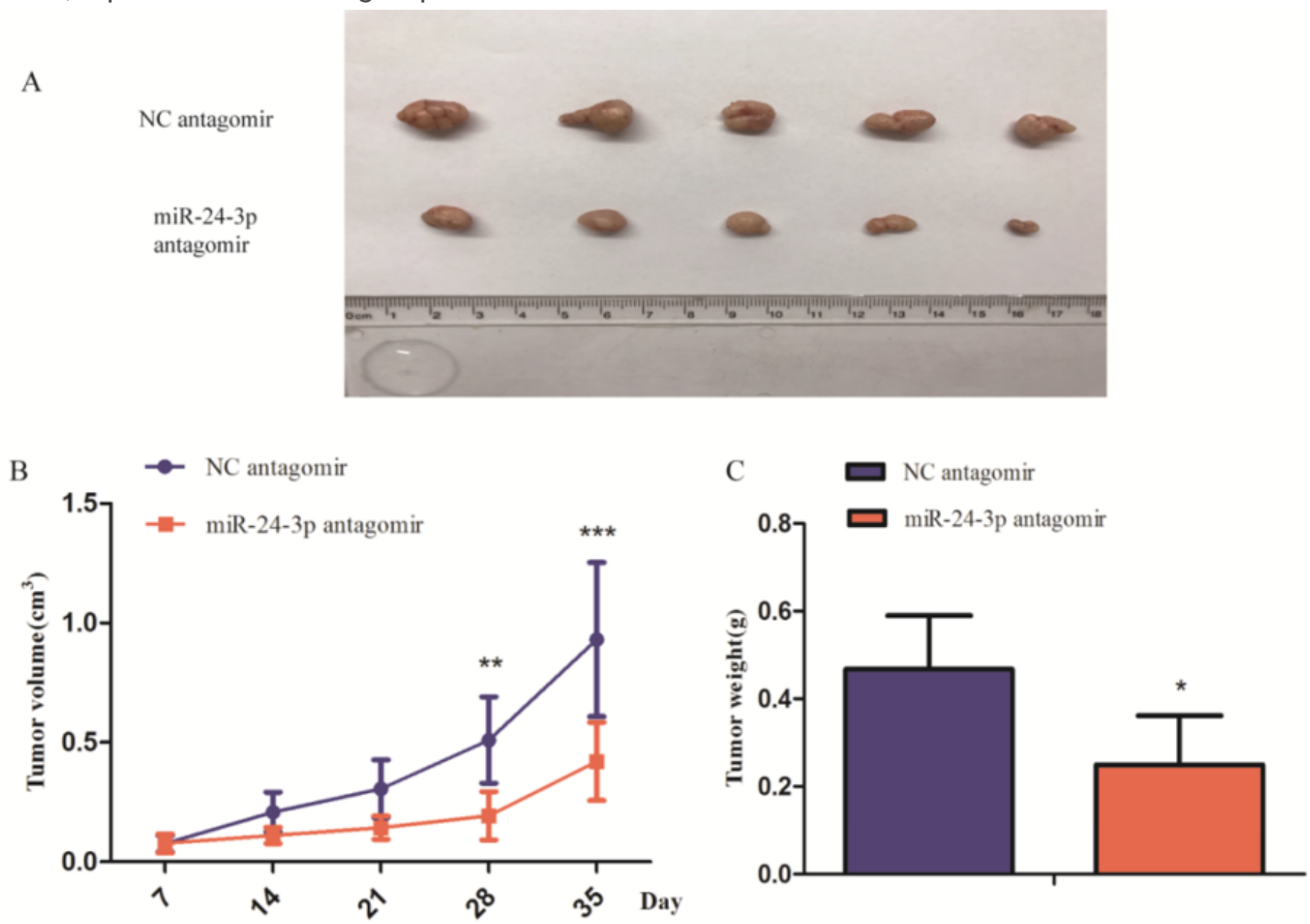

D

AMOTL2

p-YAP ${ }^{(\text {Ser 127) }}$

p-LATS 1/2
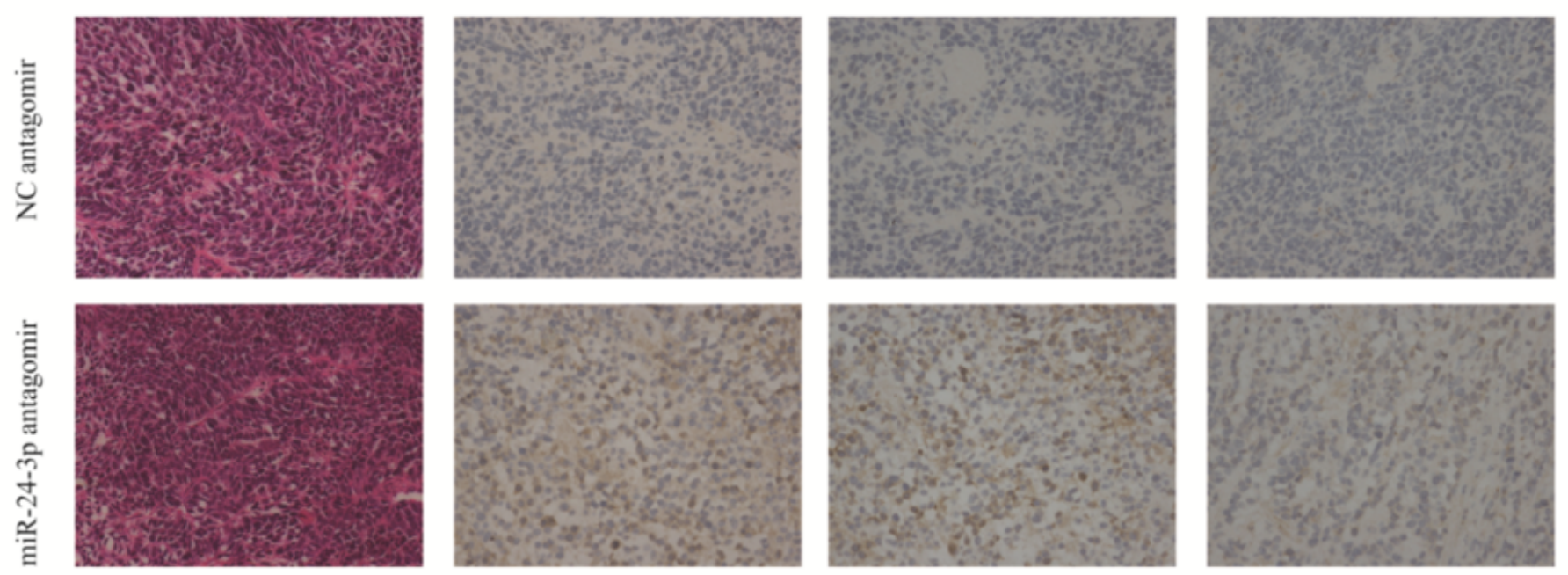

Figure 6 
miR-24-3p promoted xenograft tumor growth in vivo. (A, B, C) Mice bearing CaSki of treatment with miR24-3p antagomir subcutaneous xenografts were killed after 5 weeks (A). The tumor volumes(B) were monitored every week and tumor weights (C)were measured after the mice were sacrificed on day 35. (D) Paraffin sections were stained with hematoxylin and eosin or IHC with anti-AMOTL2, p-YAP(Ser127), and p-LATS1/2 antibodies, respectively. ${ }^{*} p<0.05,{ }^{* \star *} p<0.001$ vs. control groups.

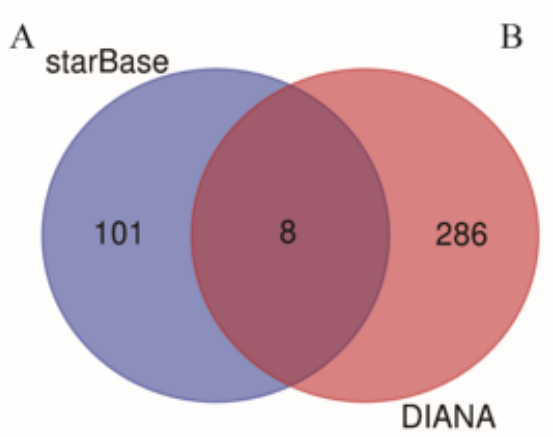

$\mathrm{C}$

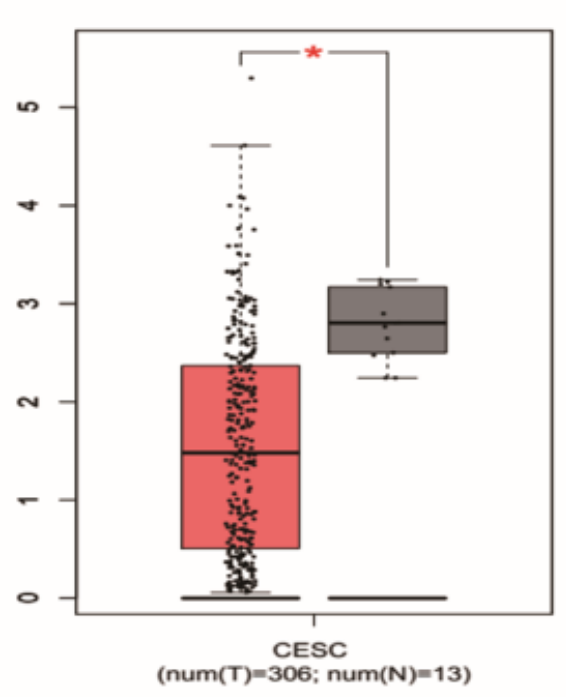

LINC00662

E

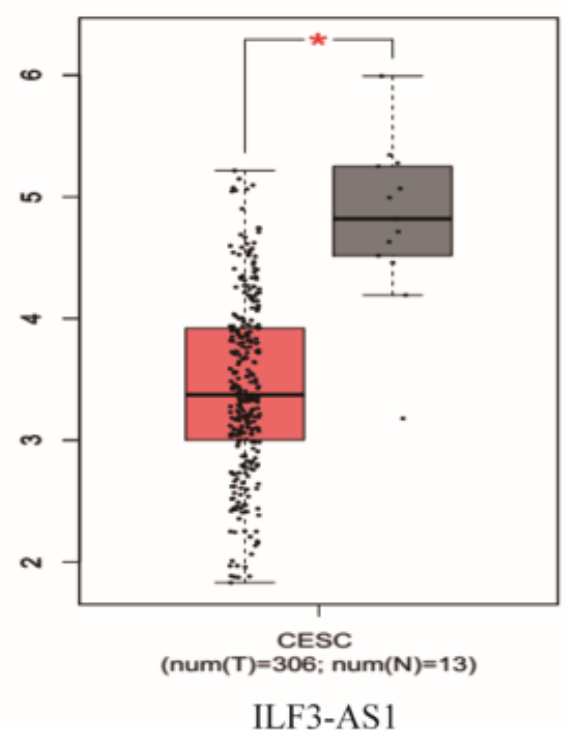

D

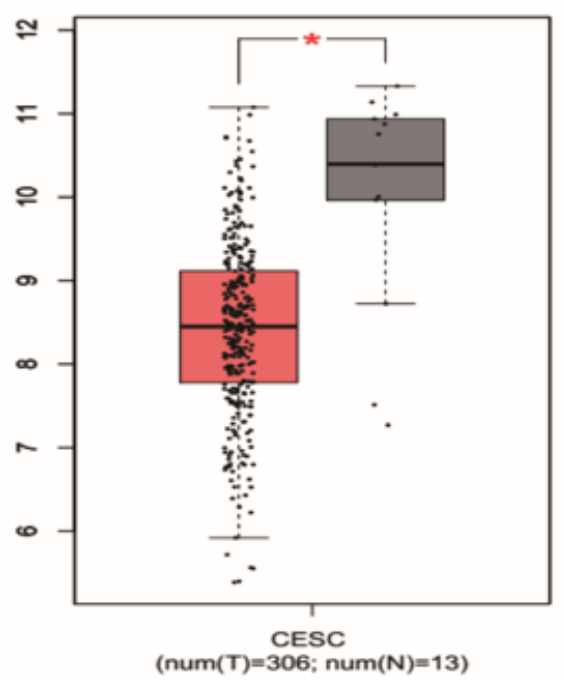

NEAT1

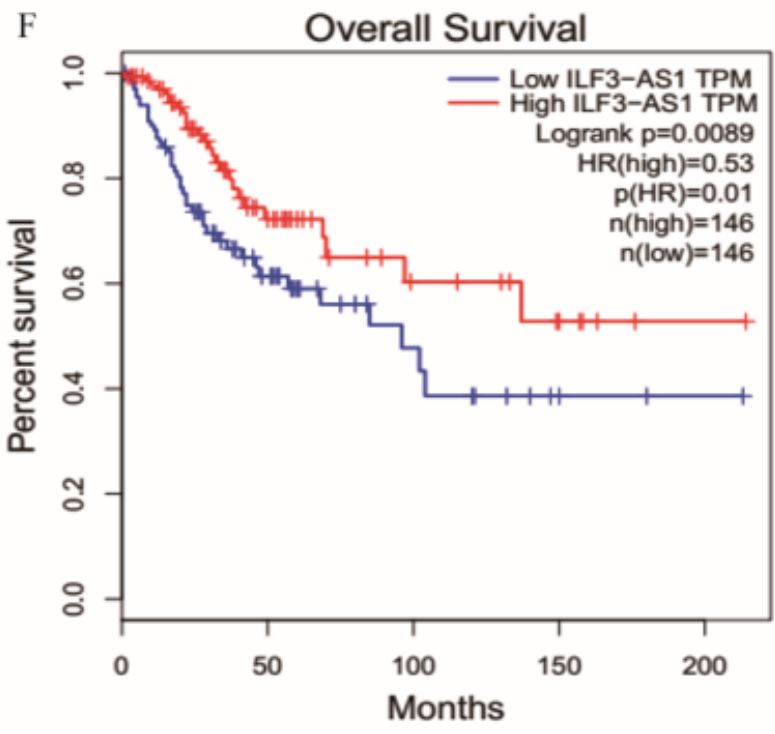

Figure 7 
Prediction and analysis of LncRNAs regulating miR-24-3p. (A) The intersection of LncRNAs was predicted by starBase and DIANA in the Venn diagram. (B) Eight LncRNAs' names in the intersection. (C, D, and E) The different expression of LINC00662, NEAT1, and ILF3-AS1 in cervical cancer and normal tissues. (F) the survival curve of ILF3-AS1 in cervical cancer. ${ }^{*} p<0.05$.

\section{Supplementary Files}

This is a list of supplementary files associated with this preprint. Click to download.

- suppfig1.png 